\title{
FRACTIONAL INTEGRATION FOR LAGUERRE EXPANSIONS
}

\section{George Gasper, Krzysztof Stempak, and Walter Trebels}

\section{Introduction}

The aim of this note is to provide a fractional integration theorem in the framework of Laguerre expansions. The method of proof consists of establishing an asymptotic estimate for the kernel involved and then applying a method of Hedberg [6]. We combine this result with the sufficient $(p, p)$ multiplier criteria of Stempak and Trebels [13]. The resulting sufficient $(p, q)$ multiplier criteria are comparable with the necessary ones of Gasper and Trebels [3,4].

Our notation is essentially that in [13]. Thus we consider the Lebesgue spaces

$$
L_{v(\gamma)}^{p}=\left\{f:\|f\|_{L_{v(\gamma)}^{p}}=\left(\int_{0}^{\infty}|f(x)|^{p} x^{\gamma} d x\right)^{1 / p}<\infty\right\}, \quad 1 \leq p<\infty, \quad \gamma>-1,
$$

and define the Laguerre function system $\left\{l_{k}^{\alpha}\right\}$ by

$$
l_{k}^{\alpha}(x)=(k ! / \Gamma(k+\alpha+1))^{1 / 2} e^{-x / 2} L_{k}^{\alpha}(x), \quad \alpha>-1, \quad k \in \mathbf{N}_{0} .
$$

This system is an orthonormal basis in $L^{2}\left(\mathbf{R}_{+}, x^{\alpha} d x\right)$ and, for $\gamma<p(\alpha+1)-1$, we can associate to any $f \in L_{v(\gamma)}^{p}$ the Laguerre series

$$
f(x) \sim \sum_{k=0}^{\infty} a_{k} l_{k}^{\alpha}(x), \quad a_{k}=\int_{0}^{\infty} f(x) l_{k}^{\alpha}(x) x^{\alpha} d x .
$$

It is convenient to introduce the vector space

$$
E=\left\{f(x)=p(x) e^{-x / 2}: 0 \leq x<\infty, \quad p(x) \text { a polynomial }\right\}
$$

which is dense in $L_{v(\gamma)}^{p}$. We note that $f \in E$ has only finitely many non-zero FourierLaguerre coefficients. Analogous to the definition of the Hardy and Littlewood fractional integral operator for Fourier series (see [15, Chap. XII, Sec. 8]), we define a fractional integral operator $I_{\sigma}, \sigma>0$, for Laguerre expansions by

$$
I_{\sigma} f(x)=\sum_{k=0}^{\infty}(k+1)^{-\sigma} a_{k} l_{k}^{\alpha}(x) \quad f \in E .
$$

Observing that the $l_{k}^{\alpha}$ are eigenfunctions with eigenvalues $\lambda_{k}$ of the differential operator

$$
L=-\left(x \frac{d^{2}}{d x^{2}}+(\alpha+1) \frac{d}{d x}-\frac{x}{4}\right), \quad \lambda_{k}=k+(\alpha+1) / 2
$$

Received November 16, 1993, revised July 8, 1994.

1991 Mathematics Subject Classification: 33C45, 42A45, 42C10.

Key words and phrases: fractional integration, multiplier conditions, Laguerre polynomials.

Research of the first author was supported in part by NSF Grant DMS-9103177.

Research of the second author was done during a stay at the Fb Mathematik of the TH Darmstadt and was supported by the Deutsche Forschungsgemeinschaft under grant 436POL/115/1/0. 
(see $[14,(5.1 .2)])$, one realizes that $I_{1}$ is an integral operator essentially inverse to $L$ (see the following Remark 2). As we will see in Section 2, the fractional integral $I_{\sigma} f$ can be interpreted as a twisted convolution of $f$ with a function

$$
g_{\sigma}(x) \sim \Gamma(\alpha+1) \sum_{k=0}^{\infty}(k+1)^{-\sigma} L_{k}^{\alpha}(x) e^{-x / 2} .
$$

From Theorem 3.1 in [4], it easily follows that $g_{\sigma} \in L_{v(\gamma)}^{1}$ when $\alpha-\gamma<\sigma$, so that, by the convolution theorem of Görlich and Markett [5], $I_{\sigma}$ extends to a bounded operator from $L_{v(\gamma)}^{p}$ to $L_{v(\gamma)}^{p}$ when $0 \leq \alpha p / 2 \leq \gamma \leq \alpha, 1 \leq p \leq \infty$. Our main result is

Theorem 1.1. Let $\alpha \geq 0,1<p \leq q<\infty$. Assume further that $0<\sigma<\alpha+1$, $a<(\alpha+1) / p^{\prime}, b<(\alpha+1) / q, a+b \geq 0$. Then

$$
\left\|I_{\sigma} f\right\|_{L_{v(\alpha-b q)}^{q}} \leq C\|f\|_{L_{v(\alpha+a p)}^{p}}, \quad \frac{1}{q}=\frac{1}{p}-\frac{\sigma-a-b}{\alpha+1} .
$$

Remarks. (1) Observe that the upper bounds for $a$ and $b$ imply lower bounds for $b$ and $a$, respectively.

(2) By the above, it is clear that the sequence $\left\{(k+1)^{-\sigma}\right\}$ which generates the fractional integral can be replaced by any sequence $\left\{\Omega_{\sigma}(k)\right\}$ satisfying

$$
\Omega_{\sigma}(k)=\sum_{j=0}^{J} c_{j}(k+1)^{-\sigma-j}+O\left((k+1)^{-\sigma-J}\right)
$$

for sufficiently large $J$, say $J \geq \alpha+2$. Thus, in particular, the same result is obtained for the sequence $\{\Gamma(k+1) / \Gamma(\sigma+k+1)\}$, see [9].

(3) A weaker version of Theorem 1.1 (e.g., in the case $a=b=0$ ) can easily be deduced by the following argument. By a slight modification of the proof of Theorem 3.1 in [4], we have:

Let $\alpha>-1$ and $N \in \mathbf{N}_{0}, N>(2 \alpha+2)(1 / r-1 / 2)-1 / 3$. If $\left\{f_{k}\right\}$ is a bounded sequence with $\lim _{k \rightarrow \infty} f_{k}=0$ and

$$
\sum_{k=0}^{\infty}(k+1)^{N+(\alpha+1) / r^{\prime}}\left|\Delta^{N+1} f_{k}\right| \leq K_{r}(f)<\infty, \quad 1 \leq r<\infty,
$$

then there exists a function $f \in L_{v(\alpha)}^{r}$ with

$$
\|f\|_{L_{v(\alpha)}^{r}} \leq C K_{r}(f), \quad f(x) \sim \sum_{k=0}^{\infty} f_{k} L_{k}^{\alpha}(x) e^{-x / 2} .
$$

This applied to the sequence $\left\{(k+1)^{-\sigma}\right\}$ gives, by Young's inequality [5],

$$
\left\|I_{\sigma} f\right\|_{L_{v(\alpha)}^{q}} \leq C\|f\|_{L_{v(\alpha)}^{p}}, \quad \frac{1}{q}>\frac{1}{p}-\frac{\sigma}{\alpha+1}
$$

where $\alpha \geq 0, \sigma>0$ and $1 \leq p, q \leq \infty$.

Next we indicate how Theorem 1.1 can be used to gain some insight into the structure of $M^{p, q}$-Laguerre multipliers. For the sake of simplicity, let us restrict ourselves 
to the case $\gamma=\alpha$. Consider a sequence $m=\left\{m_{k}\right\}$ of numbers and associate to $m$ the operator

$$
T_{m} f(x)=\sum_{k=0}^{\infty} m_{k} a_{k} l_{k}^{\alpha}(x), \quad f \in E .
$$

The sequence $m$ is called a bounded $(p, q)$-multiplier, notation $m \in M_{\alpha, \alpha}^{p, q}$, if

$$
\|m\|_{M_{\alpha, \alpha}^{p, q}}:=\inf \left\{C:\left\|T_{m} f\right\|_{L_{v(\alpha)}^{q}} \leq C\|f\|_{L_{v(\alpha)}^{p}} \text { for all } f \in E\right\}
$$

is finite. If $p \leq 2 \leq q$, then sufficient conditions follow at once in the following way: observe that $\bar{M}_{\alpha, \alpha}^{2,2}=l^{\infty}$, choose $\sigma_{0}, \sigma_{1} \geq 0$ such that $I_{\sigma_{0}}: L^{p} \rightarrow L^{2}, I_{\sigma_{1}}: L^{2} \rightarrow L^{q}$, $\sigma_{0}+\sigma_{1}=\sigma$, and hence

$$
\|m\|_{M_{\alpha, \alpha}^{p, q}} \leq\left\|k^{-\sigma_{0}}\right\|_{M_{\alpha, \alpha}^{p, 2}}\left\|\left\{k^{\sigma} m_{k}\right\}\right\|_{M_{\alpha, \alpha}^{2,2}}\left\|k^{-\sigma_{1}}\right\|_{M_{\alpha, \alpha}^{2, q}},
$$

in particular, $m \in M_{\alpha, \alpha}^{p, q}$ when $\left\{k^{\sigma} m_{k}\right\} \in l^{\infty}$. Thus, when $1<p \leq 2 \leq q<\infty$ and $\sigma$ is as in Theorem 1.1, $\left\{(-1)^{k}(k+1)^{-\sigma}\right\} \in M_{\alpha, \alpha}^{p, q}$ generates a bounded operator which does not fall under the scope of the above introduced fractional integral operators. To formulate a corollary based on a combination of Theorem 1.1 and the multiplier result in [13], we need the notion of a difference operator $\Delta^{s}$ of fractional order $s$ given by

$$
\Delta^{s} m_{k}=\sum_{j=0}^{\infty} A_{j}^{-s-1} m_{k+j}, \quad A_{j}^{t}=\frac{\Gamma(j+t+1)}{j ! \Gamma(t+1)}, \quad t \in \mathbf{R}
$$

whenever the sum converges. In view of the remark concerning the case $p<2<q$ and on account of duality, we may restrict ourselves to the case $1<p<q<2$.

Corollary 1.2. If $\alpha \geq 0,1<p<q<2$, and $s>\max \{(2 \alpha+2)(1 / q-1 / 2), 1\}$, then for some constant $C$, independent of the sequence $\left\{m_{k}\right\}$, there holds

$$
\left\|\left\{m_{k}\right\}\right\|_{M_{\alpha, \alpha}^{p, q}}^{2} \leq C\left(\left\|\left\{k^{\sigma} m_{k}\right\}\right\|_{\infty}^{2}+\sup _{N} \sum_{k=N}^{2 N}\left|(k+1)^{s+\sigma} \Delta^{s} m_{k}\right|^{2}(k+1)^{-1}\right) .
$$

The proof follows as in [2]. The result itself should be compared with the corresponding necessary condition in [3] which also shows that $\left\{(-1)^{k}(k+1)^{-\sigma}\right\} \notin M_{\alpha, \alpha}^{p, q}$ provided $1<p<q<2$ and $\alpha$ is sufficiently large.

The plan of the paper is as follows. In Section 2, we derive an asymptotic estimate of the function $g_{\sigma}$ defined above by (1). Then the twisted generalized convolution is used to dominate $I_{\sigma} f$ by a generalized Euclidean convolution of $g_{\sigma}$ with $f$. The latter's mapping behavior is discussed by a method of Hedberg [6] which uses maximal functions, thus giving Theorem 1.1 in the standard weight case $a=b=0$. In Section 3, we extend this result to some power weights, modifying an argument in Stein and Weiss $[10]$.

\section{Proof of the standard weight case}

We start by deriving the required asymptotic estimate and showing

Lemma 2.1. Let $\alpha>-1$. Then, for $x>0$ and $0<\sigma<\alpha+1$, there holds

$$
\left|g_{\sigma}(x)\right| \leq C x^{\sigma-\alpha-1} \text {. }
$$


Proof. First note that by subordination [3, p. 1234] there holds for $N>\alpha+2, N \in \mathbf{N}$,

$$
g_{\sigma}(x)=C \sum_{k=0}^{\infty}\left(\Delta^{N}(k+1)^{-\sigma}\right) L_{k}^{\alpha+N}(x) e^{-x / 2} .
$$

Then the assertion (2) follows after it is proved that

$$
\sum_{k=0}^{\infty}(k+1)^{-\sigma-N}\left|x^{\alpha+1-\sigma} L_{k}^{\alpha+N}(x) e^{-x / 2}\right| \leq C
$$

uniformly in $x>0$. With the notation

$$
\mathcal{L}_{k}^{\alpha}(x)=(k ! / \Gamma(k+\alpha+1))^{1 / 2} x^{\alpha / 2} e^{-x / 2} L_{k}^{\alpha}(x), \quad k \in \mathbf{N}_{0},
$$

this is equivalent to

$$
\sum_{k=0}^{\infty}(k+1)^{(\alpha-N) / 2-\sigma}\left|\mathcal{L}_{k}^{\alpha+N}(x)\right| \leq C x^{\sigma+(N-\alpha-2) / 2},
$$

uniformly in $0<x<\infty$. We will make use of the pointwise estimates for the Laguerre functions in [1, Sec. 2]:

$$
\left|\mathcal{L}_{k}^{\alpha+N}(x)\right| \leq C \begin{cases}(x(k+1))^{(\alpha+N) / 2}, & \text { if } \quad 0 \leq x \leq c /(k+1) \\ (x(k+1))^{-1 / 4}, & \text { if } \quad c /(k+1) \leq x \leq d(k+1)\end{cases}
$$

for fixed positive constants $c$ and $d$. These and two further estimates in $[8,(2.5)]$ imply that

$$
\sup _{x>0}\left|\mathcal{L}_{k}^{\alpha+N}(x)\right| \leq C, \quad k \in \mathbf{N}_{0}
$$

and so it is obvious that (6) implies (4) for $x \geq 1$.

Therefore, decomposing the interval $(0,1)$ dyadically, it suffices to check that

$$
\sum_{k=0}^{\infty}(k+1)^{(\alpha-N) / 2-\sigma}\left|\mathcal{L}_{k}^{\alpha+N}(x)\right| \leq C\left(2^{j}\right)^{\sigma+(N-\alpha-2) / 2}
$$

provided $2^{j} \leq x \leq 2^{j+1}, j<0$. Using the first line of (5), we get

$$
\sum_{k=0}^{2^{-j}}(k+1)^{(\alpha-N) / 2-\sigma}\left|\mathcal{L}_{k}^{\alpha+N}(x)\right| \leq C\left(2^{j}\right)^{(\alpha+N) / 2} \sum_{k=0}^{2^{-j}}(k+1)^{\alpha-\sigma} \leq C\left(2^{j}\right)^{\sigma+(N-\alpha-2) / 2}
$$

while the second line of (5) gives

$$
\sum_{k=2^{-j}}^{\infty} k^{(\alpha-N) / 2-\sigma}\left|\mathcal{L}_{k}^{\alpha+N}(x)\right| \leq C 2^{-j / 4} \sum_{k=2^{-j}}^{\infty} k^{(\alpha-N) / 2-\sigma-1 / 4} \leq C\left(2^{j}\right)^{\sigma+(N-\alpha-2) / 2} .
$$

This completes the proof of Lemma 2.1. 
As already mentioned, we will apply Hedberg's method, which involves maximal functions. To make use of the corresponding results in [11], we switch to the system

$$
\psi_{k}^{\alpha}(x)=(2 k ! / \Gamma(k+\alpha+1))^{1 / 2} e^{-x^{2} / 2} L_{k}^{\alpha}\left(x^{2}\right), \quad k \in \mathbf{N}_{0},
$$

which is obviously orthonormal on $L^{2}\left(\mathbf{R}_{+}, d \mu_{\alpha}\right), d \mu_{\alpha}(x)=x^{2 \alpha+1} d x, \alpha>-1$. For the sake of simplicity, we write the norm on $L^{p}\left(\mathbf{R}_{+}, d \mu_{\alpha}\right)$ as

$$
\|F\|_{p}=\left(\int_{0}^{\infty}|F(x)|^{p} d \mu_{\alpha}(x)\right)^{1 / p} .
$$

We adopt the notion of the twisted generalized convolution on $L^{1}\left(\mathbf{R}_{+}, d \mu_{\alpha}\right)$ from $[11,12]$,

$$
F \times G(x)=\int_{0}^{\infty} \tau_{x} F(y) G(y) d \mu_{\alpha}(y),
$$

where the twisted generalized translation operator $\tau_{x}$ is given by

$$
\tau_{x} F(y)=\frac{\Gamma(\alpha+1)}{\pi^{1 / 2} \Gamma(\alpha+1 / 2)} \int_{0}^{\pi} F\left((x, y)_{\theta}\right) \mathcal{J}_{\alpha-1 / 2}(x y \sin \theta)(\sin \theta)^{2 \alpha} d \theta,
$$

$\mathcal{J}_{\beta}(x)=\Gamma(\beta+1) J_{\beta}(x) /(x / 2)^{\beta}, J_{\beta}$ denoting the Bessel function of order $\beta$, and

$$
(x, y)_{\theta}=\left(x^{2}+y^{2}-2 x y \cos \theta\right)^{1 / 2} .
$$

With respect to the system $\left\{\psi_{k}^{\alpha}\right\}$, this convolution has the following transform property: if $F \sim \sum c_{k} \psi_{k}^{\alpha}$ and $F \times G \sim \sum c_{k} d_{k} \psi_{k}^{\alpha}$, then $G(x) \sim \Gamma(\alpha+1) \sum d_{k} L_{k}^{\alpha}\left(x^{2}\right) e^{-x^{2} / 2}$.

If we set $f\left(y^{2}\right)=F(y), g_{\sigma}\left(y^{2}\right)=G_{\sigma}(y)$, we see that

$$
\left|I_{\sigma} f\left(x^{2}\right)\right|=\left|F \times G_{\sigma}(x)\right| .
$$

Apart from Lemma 2.1, the proof of Theorem 1.1 will be based on the fact that for $\alpha \geq 0$ and suitable $F$ and $G$,

$$
|F \times G| \leq|F| *|G|
$$

which follows at once from the definition of the generalized Euclidean $*$-convolution

$$
F * G(x)=\int_{0}^{\infty} \tau_{x}^{E} F(y) G(y) d \mu_{\alpha}(y)
$$

with associated generalized Euclidean translation

$$
\tau_{x}^{E} F(y)=\int_{0}^{\pi} F\left((x, y)_{\theta}\right) d \nu_{\alpha}(\theta), \quad d \nu_{\alpha}(\theta)=\frac{\Gamma(\alpha+1)}{\pi^{1 / 2} \Gamma(\alpha+1 / 2)}(\sin \theta)^{2 \alpha} d \theta
$$

Therefore we restrict ourselves to fractional integrals defined via the generalized Euclidean convolution.

Theorem 2.2. Let $1<p<q<\infty, \alpha>-1 / 2, K_{\sigma}(x)=x^{2(\sigma-\alpha-1)}$. Then

$$
\left\|F * K_{\sigma}\right\|_{q} \leq C\|F\|_{p}, \quad \frac{1}{q}=\frac{1}{p}-\frac{\sigma}{\alpha+1} .
$$

By the above, it is clear that Theorem 1.1 for the case $a=b=0$ follows from Theorem 2.2. 
Proof. Following Hedberg [6], we want to estimate $F * K_{\sigma}(x)$ pointwise by a suitable maximal function which in this setting turns out to be (see Stempak [11, p. 138])

$$
F^{*}(x)=\sup _{\varepsilon>0} \varepsilon^{-(2 \alpha+2)} \int_{0}^{\varepsilon} \tau_{x}^{E}(|F|)(y) d \mu_{\alpha}(y)
$$

with the usual boundedness property $\left\|F^{*}\right\|_{r} \leq C\|F\|_{r}, 1<r \leq \infty$. Now there holds

$$
\left|F * K_{\sigma}(x)\right| \leq C\left(\int_{0}^{\delta}+\int_{\delta}^{\infty}\right) \tau_{x}^{E}(|F|)(y) y^{2(\sigma-\alpha-1)} d \mu_{\alpha}(y)=J_{1}+J_{2},
$$

where $\delta>0$ will be chosen later appropriately. Clearly,

$$
\begin{aligned}
J_{1} & =\sum_{k=0}^{\infty} \int_{2^{-k-1} \delta}^{2^{-k} \delta} \cdots \leq C \sum_{k=0}^{\infty}\left(2^{-k} \delta\right)^{2 \sigma}\left(2^{-k} \delta\right)^{-2 \alpha-2} \int_{2^{-k-1} \delta}^{2^{-k} \delta} \tau_{x}^{E}(|F|)(s) s^{2 \alpha+1} d s \\
& \leq C \delta^{2 \sigma} \sum_{k=0}^{\infty} 2^{-k 2 \sigma}\left(2^{-k} \delta\right)^{-2 \alpha-2} \int_{0}^{\delta 2^{-k}} \tau_{x}^{E}(|F|)(s) s^{2 \alpha+1} d s \leq C \delta^{2 \sigma} F^{*}(x) .
\end{aligned}
$$

On the other hand, by Hölder's inequality,

$$
\begin{aligned}
J_{2}=\int_{\delta}^{\infty} \tau_{x}^{E}(|F|)(s) s^{2(\sigma-\alpha-1)} s^{2 \alpha+1} d s & \leq\left\|\tau_{x}^{E}(|F|)\right\|_{p}\left(\int_{\delta}^{\infty} s^{2(\sigma-\alpha-1) p^{\prime}} s^{2 \alpha+1} d s\right)^{1 / p^{\prime}} \\
& \leq C \delta^{2 \sigma-(2 \alpha+2) / p}\|F\|_{p}
\end{aligned}
$$

since $\tau_{x}^{E}$ are contractions on $L^{p}\left(\mathbf{R}_{+}, d \mu_{\alpha}\right)$. Hence

$$
\left|F * K_{\sigma}(x)\right| \leq C\left(\delta^{2 \sigma} F^{*}(x)+\delta^{2 \sigma-(2 \alpha+2) / p}\|F\|_{p}\right) .
$$

Choosing $\delta=\left(F^{*}(x) /\|F\|_{p}\right)^{-p /(2 \alpha+2)}$ (where $\|F\|_{p} \neq 0$ is assumed), we obtain

$$
\left|F * K_{\sigma}(x)\right| \leq C F^{*}(x)^{1-\sigma p /(\alpha+1)}\|F\|_{p}^{\sigma p /(\alpha+1)}
$$

and then $\left\|F * K_{\sigma}\right\|_{q} \leq C\|F\|_{p}$ due to the inequality for the maximal function with $r=q(1-\sigma p /(\alpha+1))=p>1$. This finishes the proof of Theorem 2.2.

\section{Extension to power weights}

The proof of Theorem 1.1 in the general case follows along the lines in Section 2 from

Theorem 3.1. Let $\alpha>-1 / 2,0<\sigma<\alpha+1$ and $a<(\alpha+1) / p^{\prime}, b<(\alpha+1) / q$, with $a+b \geq 0$. If $1<p \leq q<\infty$, then

$$
\left\|K_{\sigma} * F(x) x^{-2 b}\right\|_{q} \leq C\left\|F(x) x^{2 a}\right\|_{p}, \quad \frac{1}{q}=\frac{1}{p}-\frac{\sigma-a-b}{\alpha+1} .
$$

Proof. An equivalent version of the inequality above is

$$
\left(\int_{0}^{\infty}|S f(x)|^{q} d \mu_{\alpha}(x)\right)^{1 / q} \leq C\left(\int_{0}^{\infty}|f(x)|^{p} d \mu_{\alpha}(x)\right)^{1 / p}
$$

where

$$
f(x)=x^{2 a} F(x), \quad S f(x)=\int_{0}^{\infty} K(x, y) f(y) d \mu_{\alpha}(y)
$$


and

$$
K(x, y)=x^{-2 b}\left(\int_{0}^{\pi}(x, y)_{\theta}^{2(\sigma-\alpha-1)} d \nu_{\alpha}(\theta)\right) y^{-2 a} .
$$

We first consider the case $p=q$. Then $\sigma=a+b$ and, therefore, the kernel $K(x, y)$ is homogeneous of degree $-(2 \alpha+2): K(r x, r y)=r^{-(2 \alpha+2)} K(x, y), r>0$. It now suffices to check that (cf. Section 2 of [13])

$$
\int_{0}^{\infty} K(1, y) y^{-(2 \alpha+2) / p} d \mu_{\alpha}(y)<\infty
$$

We first note that the function $K(1, y)$ has at most an integrable singularity at $y=1$, since for $1 / 2 \leq y \leq 2$ we have

$$
\int_{0}^{\pi} \frac{(\sin \theta)^{2 \alpha} d \theta}{\left((1-y)^{2}+4 y \sin ^{2}(\theta / 2)\right)^{\alpha+1-\sigma}} \leq C\left(1+|1-y|^{\sigma-1}\right) .
$$

To deal with the singularity at 0 , we note that for $y<1 / 2$ we have $(1, y)_{\theta} \approx 1$, and therefore $\int_{0}^{\pi}(1, y)_{\theta}^{2(\sigma-\alpha-1)} d \nu_{\alpha}(\theta) \approx 1$ as well. Hence $K(1, y) \leq C y^{-2 a}$ and thus

$$
\int_{0}^{1 / 2} K(1, y) y^{-(2 \alpha+2) / p} d \mu_{\alpha}(y)<\infty, \quad a<(\alpha+1) / p^{\prime} .
$$

For $y>2$, we have $(1, y)_{\theta} \approx y, \int_{0}^{\pi}(1, y)_{\theta}^{2(\sigma-\alpha-1)} d \nu_{\alpha}(\theta) \approx y^{2(\sigma-\alpha-1)}$, and hence

$$
\int_{2}^{\infty} K(1, y) y^{-(2 \alpha+2) / p} d \mu_{\alpha}(y)<\infty, \quad b<(\alpha+1) / q
$$

since $\sigma<(\alpha+1) / p$.

Now, consider the case $1<p<q<\infty$. We use still another equivalent version of the inequality to be proved, namely

$$
\int_{0}^{\infty} \int_{0}^{\infty} K(x, y) f(y) g(x) d \mu_{\alpha}(y) d \mu_{\alpha}(x) \leq C\|f\|_{p}\|g\|_{q^{\prime}}
$$

assuming for simplicity that $f$ and $g$ are nonnegative. Writing $\mathbf{R}_{+} \times \mathbf{R}_{+}=D_{1} \cup D_{2} \cup D_{3}$ where

$$
D_{1}=\{y / 2 \leq x \leq 2 y\}, \quad D_{2}=\{2 y<x\}, \quad D_{3}=\{x<y / 2\},
$$

it suffices to check for $i=1,2,3$ that

$$
I_{i}=\iint_{D_{i}} K(x, y) f(y) g(x) d \mu_{\alpha}(y) d \mu_{\alpha}(x) \leq C\|f\|_{p}\|g\|_{q^{\prime}}
$$

Consider $I_{1}$ first. Since $a+b \geq 0$, for $x, y \in D_{1}$ and $\theta \in(0, \pi)$ we have

$$
\left((x, y)_{\theta}\right)^{2(a+b)} \leq C x^{2(a+b)} \leq C x^{2 b} y^{2 a}
$$

so

$$
I_{1} \leq \iint_{D_{1}} \tilde{K}(x, y) f(y) g(x) d \mu_{\alpha}(y) d \mu_{\alpha}(x)
$$

with $\widetilde{K}(x, y)=\tau_{x}^{E} K_{\sigma-a-b}(y)$. Hence we are reduced to showing that

$$
\int_{0}^{\infty} K_{\sigma-a-b} * f(x) g(x) d \mu_{\alpha}(x) \leq\|f\|_{p}\|g\|_{q^{\prime}},
$$

which is implied by Theorem 2.2 . 
To estimate $I_{2}$ and $I_{3}$, we need the following lemma.

Lemma 3.2. Let $\alpha>-1$ and $V_{\delta} f(x)=x^{2(\delta-\alpha-1)} \int_{0}^{x} f(y) y^{-2 \delta} d \mu_{\alpha}(y), \delta<(\alpha+1) / p^{\prime}$. Then

$$
\left\|V_{\delta} f\right\|_{p} \leq C\|f\|_{p}, \quad\left|V_{\delta} f(x)\right| \leq C x^{-(2 \alpha+2) / p}\|f\|_{p} .
$$

Proof. We have that $V_{\delta} f(x)=\int_{0}^{\infty} L(x, y) f(y) d \mu_{\alpha}(y)$, where $L(x, y)=x^{2(\delta-\alpha-1)} y^{-2 \delta}$ for $y<x$ and equals 0 otherwise. Clearly $L$ is homogeneous of degree $-(2 \alpha+2)$. Therefore the norm inequality is implied by

$$
\int_{0}^{\infty} L(1, y) y^{-(2 \alpha+2) / p} d \mu_{\alpha}(y)=\int_{0}^{1} y^{-2 \delta-(2 \alpha+2) / p+2 \alpha+1} d y<\infty,
$$

which is finite since $\delta<(\alpha+1) / p^{\prime}$. For the pointwise estimate, we simply write

$$
\begin{aligned}
\left|V_{\delta} f(x)\right| & \leq x^{2(\delta-\alpha-1)} \int_{0}^{x}|f(y)| y^{-2 \delta} d \mu_{\alpha}(y) \\
& \leq C x^{2(\delta-\alpha-1)}\left(\int_{0}^{x} y^{-2 \delta p^{\prime}} d \mu_{\alpha}(y)\right)^{1 / p^{\prime}}\|f\|_{p} \\
& \leq C x^{-(2 \alpha+2) / p}\|f\|_{p},
\end{aligned}
$$

which finishes the proof of the lemma.

Estimating $I_{2}$, we note that for $x, y \in D_{2}$ and all $\theta, 0<\theta<\pi$, there holds $x<2(x-y) \leq 2\left((x-y)^{2}+2 x y(1-\cos \theta)\right)^{1 / 2}=2(x, y)_{\theta}$. Hence

$$
\begin{aligned}
I_{2} & \leq C \int_{0}^{\infty} x^{2(\sigma-\alpha-1-b)} g(x)\left(\int_{0}^{x} f(y) y^{-2 a} d \mu_{\alpha}(y)\right) d \mu_{\alpha}(x) \\
& =C \int_{0}^{\infty} g(x) x^{2(\sigma-a-b)} V_{a} f(x) d \mu_{\alpha}(x) \leq C\|g\|_{q^{\prime}}\left\|x^{2(\sigma-a-b)} V_{a} f\right\|_{q} .
\end{aligned}
$$

It now suffices to estimate the latter $L^{q}$ norm by $\|f\|_{p}$. We have

$$
\int_{0}^{\infty}\left|x^{2(\sigma-a-b)} V_{a} f(x)\right|^{q} d \mu_{\alpha}(x)=\int_{0}^{\infty}\left|V_{a} f(x)\right|^{p}\left|V_{a} f(x)\right|^{q-p} x^{2(\sigma-a-b) q} d \mu_{\alpha}(x) .
$$

Since $a<(\alpha+1) / p^{\prime}$, by the pointwise estimate in the above lemma

$$
\left|V_{a} f(x)\right| \leq C x^{-(2 \alpha+2) / p}\|f\|_{p},
$$

hence

$$
\left|V_{a} f(x)\right|^{q-p} x^{2(\sigma-a-b) q} \leq C\|f\|_{p}^{q-p}
$$

due to the identity from assumptions. Hence

$$
\int_{0}^{\infty}\left|x^{2(\sigma-a-b)} V_{a} f(x)\right|^{q} d \mu_{\alpha}(x) \leq C\|f\|_{p}^{q-p} \int_{0}^{\infty}\left|V_{a} f(x)\right|^{p} d \mu_{\alpha}(x) \leq C\|f\|_{p}^{q}
$$

by the norm inequality from Lemma 3.2. The estimate of $I_{3}$ is similar and we omit it. The proof of the theorem is complete.

After submitting this paper the authors became acquainted with the following result of Kanjin and Sato [7]:

$$
\left\|I_{\sigma} f\right\|_{L_{v(\alpha q / 2)}^{q}} \leq C\|f\|_{L_{v(\alpha p / 2)}^{p}}, \quad 0<\frac{1}{q}=\frac{1}{p}-\sigma, \quad p>1, \quad \alpha \geq 0,
$$


whereas for $-1<\alpha<0$, there occurs $\left(1+\frac{\alpha}{2}\right)^{-1}<p, q<-\frac{2}{\alpha}$ as an additional restriction. Combining the above Theorem 1.1 in the case $a=b=\alpha=0$ with Kanjin's transplantation theorem (cf. [13]) one at once recovers the above stated result of Kanjin and Sato.

\section{References}

1. R. Askey and S. Wainger, Mean convergence of expansions in Laguerre and Hermite series, Amer. J. Math. 87 (1965), 695-708.

2. G. Gasper and W. Trebels, Jacobi and Hankel multipliers of type $(p, q), 1<p<q<\infty$, Math. Ann. 237 (1978), 243-251.

3. __ On necessary multiplier conditions for Laguerre expansions, Canad. J. Math. 43 (1991), $1228-1242$.

4. _ On necessary multiplier conditions for Laguerre expansions II, SIAM J. Math. Anal. 25 (1994), 384-391.

5. E. Görlich and C. Markett, A convolution structure for Laguerre series, Indag. Math. 44 (1982), 161-171.

6. L. I. Hedberg, On certain convolution inequalities, Proc. Amer. Math. Soc. 36 (1972), 505-510.

7. Kanjin and Sato, The Hardy-Littlewood theorem on fractional integration for Laguerre series, Proc. Amer. Math. Soc. (to appear).

8. B. Muckenhoupt, Mean convergence of Hermite and Laguerre series II, Trans. Amer. Math. Soc. 147 (1970), 433-460.

9. - Transplantation theorems and multiplier theorems for Jacobi series, Mem. Amer. Math. Soc. 64 (1986), No. 356.

10. E. M. Stein and G. Weiss, Fractional integrals on n-dimensional Euclidean space, J. Math. Mech. 7 (1958), 503-514.

11. K. Stempak, Almost everywhere summability of Laguerre series, Studia Math. 100 (1991), 129147.

12. ___ Almost everywhere summability of Laguerre series II, Studia Math. 103 (1992), 317-327.

13. K. Stempak and W. Trebels, On weighted transplantation and multipliers for Laguerre expansions, Math. Ann. 300 (1994), 203-219.

14. G. Szegö, Orthogonal Polynomials, 4th ed., Amer. Math. Soc. Colloq. Publ. 23, Providence, R.I., 1975.

15. A. Zygmund, Trigonometric Series, 2nd ed., Cambridge Univ. Press, Cambridge 1968.

Dept. of Mathematics, Northwestern University, Evanston, IL 60208, U.S.A.

Institute of Mathematics, University of Wroceaw, Pl. Grunwaldzki 2/4, 50-384 Wroceaw, POLAND

Fb. Mathematik, Th Darmstadt, Schlossgartenstr. 7, D-64289 Darmstadt, Germany 